\title{
http://dx.doi.org/10.35381/r.k.v4i2.468
}

\section{El examen especial como proceso de control: fases y aplicación práctica en el sector público}

\section{Special examination as a control process: phases and practical application in the public sector}

\author{
Diana Paulina Figueroa Hurtado \\ dfigueroah@psg.ucacue.edu.ec \\ Universidad Católica de Cuenca, Cuenca \\ Ecuador \\ https://orcid.org/0000-0002-9706-4865 \\ Cecilia Ivonne Narváez Zurita \\ inarvaez@ucacue.edu.ec \\ Universidad Católica de Cuenca, Cuenca \\ Ecuador: \\ https://orcid.org/0000-0002-7437-9880 \\ Juan Carlos Erazo Álvarez \\ jcerazo@ucacue.edu.ec \\ Universidad Católica de Cuenca, Cuenca \\ Ecuador: \\ https://orcid.org/0000-0001-6480-2270
}

Recibido: 1 de septiembre de 2019

Aprobado: 30 de septiembre de 2019

\section{RESUMEN}

El examen especial (EE) permite detectar hallazgos en las instituciones auditadas, además analiza la eficiencia, eficacia, efectividad y economía en la administración. El presente artículo científico se basa en la problemática de como evaluar la administración de talento humano del Registro de la Propiedad del cantón Cuenca; para lo cual se ha planteado como objetivo la aplicación de un EE al proceso administrativo en el período enero a diciembre de 2018, con fines de verificación del cumplimiento de este, de acuerdo a la normativa vigente. Se aplicó un estudio de tipo experimental, ya que se ejecutó un EE para identificar el cumplimiento de los procesos; con lo que se pudo determinar que la entidad no cumple de manera íntegra la ejecución del programa de capacitación y de 
igual manera inobserva la normativa referente a la presentación del reporte de las declaraciones patrimoniales a la Contraloría General del Estado Ecuatoriano.

Descriptores: Auditoría; Examen especial; Control interno; Talento humano; Riesgos.

\begin{abstract}
The special examination (EE) allows to detect findings in the audited institutions, in addition the efficiency, accuracy, effectiveness and economy in the administration are analyzed. This scientific article is based on the problem of how to evaluate the administration of human talent of the Registro de la Propiedad of the canton Cuenca; for which purpose the application of an EE to the administrative process in the period from january to december 2018 has been considered, with the purpose of verifying compliance with it, in accordance with current regulations. An experimental study was applied, since a EE was executed to identify the compliance of the processes; with what could be determined that the entity does not fully comply with the execution of the training program and in the same way does not comply with the regulations regarding the presentation of the report of the capital declarations to the Government Accountability Office.
\end{abstract}

Descriptors: Audit; Special exam; Internal control; Human talent; Risks.

\title{
INTRODUCCIÓN
}

El examen especial (EE) verifica, analiza y evalúa determinados procesos concernientes a la gestión de la organización; se realiza de manera posterior a la ejecución de los hechos y se concluye con el correspondiente informe que debe contener comentarios, conclusiones y recomendaciones (Congreso Nacional, 2002).

Según lo que dispone la Constitución de la República del Ecuador, los servidores del sector público deberán cumplir únicamente con las funciones dispuestas en la ley (Constitución de la República del Ecuador, 2008). Por ende, considerando que las entidades de este sector manejan recursos del Estado, deben basar su accionar en lo que dispone la Contraloría General del Estado (CGE), ya que es el ente que controla el uso de recursos públicos y dentro de sus competencias está la determinación de responsabilidades administrativas, civiles e indicios de responsabilidad penal; las mismas que se establecen al realizar los EE por parte del ente de control (Constitución de la República 
del Ecuador, 2008).

\section{DESARROLLO}

Desde la edad media hasta la revolución industrial se realizaban auditorías con el fin de verificar si los empleados del gobierno se desempeñaban con honestidad. En la primera mitad del siglo XX, el trabajo de auditoría se centró en determinar si los estados financieros proporcionaban una real situación financiera, en lugar de descubrir fraudes. Los auditores debían dar cuentas a las oficinas gubernamentales y no sólo a los accionistas. Con el surgimiento de compañías en Estados Unidos y en Gran Bretaña, los auditores escogieron algunas transacciones para analizarlas y llegaron a la conclusión de que examinar pocas transacciones proporciona confiabilidad de otras transacciones semejantes, sin la necesidad de evaluarlas a todas (Whittington y Pany, 2005).

Además del muestreo los auditores consideraron la importancia de mantener un eficiente control interno, este proceso consiste en cumplir con la normativa que rige a la entidad o empresa, con el fin de alcanzar los objetivos planteados. El análisis del control interno permite identificar las áreas que tienen fortaleza y debilidades. Al aplicar el muestreo y el control interno, se evidenció la dificultad de algunos auditores para detectar el fraude. A partir de la década de 1960 la detección de fraudes asumió un papel más importante en el proceso de auditoría. A raíz de la detección de varios casos de fraude, las principales organizaciones contables patrocinaron la National Commission on Fraudulent Financial Reporting para que estudiara las causas e hiciera recomendaciones para disminuir este problema.

En el año 2000 a solicitud de la Securities and Exchange Commission, se creó el Panel on Audit Effectiveness, que se encargaba de evaluar cómo se realizaban las auditorías independientes de los estados financieros; a raíz de las recomendaciones del panel se hicieron cambios en las normas referentes a la detección de fraudes. A partir de diciembre de 2001, algunas compañías públicas reformularon los estados financieros del período anterior, lo que provocó la desconfianza de los inversionistas y agitó un mercado 
ya débil desde finales del 2001 e inicios del 2002; cuestionando la eficacia de las auditorías de los estados financieros por lo que restó credibilidad a la profesión contable (Whittington y Pany, 2005).

Bajo este contexto, Tapia, Guevara, Castillo, Rojas y Salomón (2016) señalan que la auditoría consiste en revisar que los hechos se desarrollen, en la forma que fueron planteados y cumpliendo con la normativa establecida. Por su parte, Franklin (2013) infiere que una auditoría administrativa es la revisión que se realiza al interior de un organismo, con la finalidad de determinar su nivel de desempeño y definir estrategias que permitan mejorar y conlleven a lograr una ventaja competitiva.

La Norma Internacional ISO 19011 (2011) establece que la auditoría se caracteriza por depender de varios principios, que permiten que la misma sea una herramienta eficaz y fiable que apoye a los controles de la gestión. Estos son: integridad que se refiere a que los auditores al desarrollar su trabajo de auditoría, deben realizarlo con honestidad, diligencia, imparcialidad y responsabilidad cumpliendo con toda la normativa legal; presentación ecuánime que se basa en la obligación que tienen los auditores de comunicar de manera veraz y exacta los hallazgos, conclusiones e informes de la auditoría; debido cuidado profesional, es decir los profesionales deben actuar con el esmero que la actividad que desarrollan lo amerita y de esa manera retribuir la confianza que han depositado en ellos, emitiendo el informe con un juicio razonable; confidencialidad que se refiere a que los auditores deben ser discretos con la información obtenida, la misma no puede ser utilizada en perjuicio del auditado; independencia que se basa en que la auditoría sea ejecutada de manera imparcial y para lograrlo los auditores no deben tener relación con la entidad examinada; enfoque basado en la evidencia que es el método racional que nos permite alcanzar conclusiones fiables, dentro del proceso de auditoría, basándonos en la evidencia encontrada.

\section{Auditoría gubernamental}

Es la evaluación ecuánime, sistematizada, imparcial y que se efectúa a la gestión de la organización; mediante la cual se puede recabar evidencias que permitan verificar que 
los recursos del Estado hayan sido administrados de manera eficiente, efectiva, eficaz, económica y transparente (CGE, 2003).

Según dispone el Reglamento de la Ley Orgánica de la Contraloría General del Estado, el control mediante la auditoría se aplicará a todas las instituciones señaladas en el Art. 118 de la Constitución del Ecuador, incluyendo a las entidades financieras públicas, en tal virtud para ejecutar una auditoría gubernamental se deberá planificar y evaluar posteriormente sus resultados. En este tipo de auditoría no existen soluciones definidas a seguir, por lo que el auditor debe guiarse por su alto espíritu de servicio y su juicio profesional (Sotomayor, 2008).

La Organización Internacional de Entidades Fiscalizadoras Superiores INTOSAI (2013) señala que el entorno de la auditoría del sector público, es aquel en donde las entidades de este sector y aquellas del sector privado que reciben recursos públicos, son responsables de los recursos percibidos por lo que deben rendir cuentas sobre el uso de estos y de su gestión; ya que se evalúa su desempeño según las leyes y reglamentos aplicables. Entre algunos de los principios que la INTOSAl establece para la auditoría en el sector público, están: gestión y habilidades del equipo de auditoría, este principio indica que quienes conformen el equipo de auditoría deben poseer conocimientos, habilidades y la experiencia necesaria que les permita concluir exitosamente la misma; riesgo de auditoría en donde los auditores deben manejar el riesgo de proporcionar un informe que sea inapropiado, a fin de reducir la contingencia de llegar a conclusiones inadecuadas; materialidad que se refiere a que un asunto se lo puede considerar importante si el conocimiento de este influye en las decisiones de los usuarios; y, comunicación, este principio se refiere a que durante el proceso de auditoría es importante que se mantenga informada a la entidad auditada.

\section{Alcance y modalidades de auditoría gubernamental}

La Ley Orgánica de la Contraloría General del Estado establece, que el control que realiza el ente regulador, lo ejerce a través de auditorías gubernamentales y exámenes especiales. La auditoría gubernamental examina el accionar de los administradores de los 
fondos estatales y el examen especial evalúa aspectos limitados de las actividades relativas a la gestión financiera, administrativa, operativa y medio ambiental posteriormente a su ejecución (Congreso Nacional, 2002). Los tipos de auditoría gubernamental según Congreso Nacional (2002) y Fonseca (2007) son: auditoría financiera, auditoría de gestión y el examen especial.

Auditoría financiera. -Tiene por objetivo determinar si los estados financieros del ente auditado presentan razonablemente su situación financiera; este tipo de auditoría permite encontrar posibles falencias y plasmarlas en el informe.

Auditoría de gestión. - Permite evaluar el manejo de los recursos públicos, así como el desempeño de los servidores y funcionarios del Estado; con el fin de determinar si este se ha logrado basándose en criterios eficaces, eficientes, económicos y efectivos.

Examen especial. - Esta auditoría puede combinar a la auditoría financiera con la auditoría de gestión y verifica aspectos limitados del manejo de los recursos públicos en un período determinado; así como al cumplimiento de las disposiciones legales aplicables. En Ecuador el EE es realizado por la CGE, de manera posterior a la ejecución de los procesos de la entidad auditada; al concluir dicho examen se podrá determinar responsabilidades de tipo administrativas, civiles culposas e indicios de responsabilidad penal, por acción u omisión en contra de las disposiciones legales o por perjuicio económico a los recursos públicos, contando con la evidencia necesaria.

En este sentido, Blanco (2012) señala que la evidencia se obtiene de una mezcla de pruebas de control, de procedimientos sustantivos y análisis de los indicadores claves, en tanto que, Pucha, Narváez, Erazo y Torres (2019) refieren que los papeles son la evidencia del trabajo ejecutado por el auditor y sirven de soporte en el informe final. Los papeles de trabajo permiten demostrar los hechos encontrados.

\section{Fases del proceso del EE}

Planificación: en la planificación el auditor identifica los procesos más críticos, los potenciales problemas, evalúa el nivel de riesgo y confianza y elabora un programa a seguir para obtener la evidencia necesaria; a fin de revisar los procedimientos de la institución 
examinada. El Manual General de Auditoría Gubernamental (2003) refiere que el alcance de tiene la planificación puede presentar variaciones, dependiendo la magnitud de las operaciones de la institución, el tamaño de la misma y la experticia del auditor.

Esta etapa requiere de dedicación y que todo el equipo de auditoría llegue a un consenso, que les permita identificar el objetivo y el alcance que tengan previsto otorgarle; no obstante, se pueden producir cambios en el desarrollo de la auditoría. Con esto se espera que la planeación haga posible que todo el proceso de auditoria se realice de manera eficaz y eficiente, garantizando así la consecución de los objetivos planteados (Sotomayor, 2008).

Franklin (2013) infiere que planear es definir el camino que se recorrerá durante la auditoría. En esta fase se integra los objetivos, instrumentos y métodos, haciendo posible un nexo entre los fines que se persigue alcanzar y los medios empleados para lograrlos.

En este proceso el auditor identifica las áreas y problemas que serán materia del análisis, se programa la obtención de la evidencia, se define la estrategia a utilizar y se integran los objetivos y los métodos de tal manera que se cree un vínculo entre los propósitos que se persiguen y los medios que se utilizarán para alcanzarlos. Dentro de la planificación en el sector gubernamental se identifica: la planificación preliminar y la planificación específica.

Planificación preliminar. - Esta fase empieza con la orden de trabajo; aquí se realizará una guía para la visita previa, a fin de obtener información de la institución a ser auditada. En este conocimiento preliminar se identificarán los siguientes aspectos como la visión, la misión, los objetivos, la naturaleza jurídica, la base legal de la institución, entre otros (CGE, 2003; CGE, 2011).

Al finalizar esta etapa se debe presentar un informe, el mismo que proporciona una perspectiva de la auditoría a ejecutarse a fin de determinar los componentes a ser evaluados en la siguiente fase, respaldado en los papeles de trabajo; se puede exceptuar la presentación de este informe al tratarse de entidades pequeñas (CGE, 2003). En esta fase gracias a la experiencia del auditor, el diagnóstico parte de la percepción que se forma este, 
sobre la realidad y la cultura de la organización; la cual es intangible, sin embargo es posible percibirla en el desempeño del trabajo (Franklin, 2013).

Planificación específica.- Se fundamenta en los datos recopilados en la etapa preliminar y consiste en la verificación de hechos, a través de esta información y mediante entrevistas a funcionarios; su objetivo es identificar las actividades relevantes de la entidad auditada para examinarlas en la siguiente fase y cuyo propósito es realizar la evaluación de control interno, calificar los riesgos de la auditoría y seleccionar los procedimientos que se aplicarán en la fase de ejecución (CGE, 2011).

En lo que respecta a los riesgos, existen el riesgo inherente que es el que está intrínseco en la naturaleza de cada actividad; el riesgo de control, es el riesgo de no obtener la eficacia esperada, con el sistema de control interno aplicado al interior de la entidad y el riesgo de detección que es el riesgo de obtener un informe de auditoría errado, por la probabilidad que el auditor no haya detectado alguna inobservancia en el proceso del examen (Estupiñan, 2015).

El grado de planeación dependerá del tamaño de la entidad, la complejidad de la auditoría y la experiencia del auditor (Instituto Mexicano de Contadores Públicos, 2002).

La planificación dentro de la auditoría gubernamental sirve al auditor como una guía de los procesos a revisar, con el fin de verificar el uso correcto de los recursos públicos. Franklin (2013) dice, la planificación consiste en determinar las acciones que se van a desarrollar en forma secuencial y ordenada, con el fin de optimizar el tiempo y alcanzar los objetivos establecidos.

Al tratarse de un examen que se ejecuta por primera vez en una institución la información que se obtendrá será numerosa, al contrario, cuando se trata de exámenes concurrentes, la información se deberá actualizar desde el examen anterior (CGE, 2011).

Considerando que el propósito de la planificación específica es evaluar el control interno y esta evaluación está direccionada a los procesos vigentes, se aplican los componentes de control interno: ambiente de control, evaluación del riesgo, actividades de control, información y comunicación y seguimiento; con la finalidad de determinar si la entidad ejecutó sus competencias de manera eficaz, eficiente, efectiva y económica. 
El control interno comprende el plan que adopte la organización para proteger sus activos, verificar la exactitud y la confiabilidad de sus datos contables, promover la eficiencia operacional y fomentar el cumplimiento de la normativa que les regula (Mantilla, 2018). El control interno es un proceso preventivo, que engloba a todas las actividades de las organizaciones y brinda una seguridad razonable de que los procesos están encaminados a la consecución de los objetivos; además garantiza el cumplimiento del marco jurídico y el buen uso de los recursos (Portal, 2016).

Las instituciones del sector público y entidades del sector privado del Ecuador que reciban recursos públicos, deben regir sus actividades por las normas de control interno aplicables para este sector; las mismas que están emitidas en base a los cinco componentes del control interno, mencionados anteriormente.

En base a los resultados de la evaluación del sistema de control interno, se determinarán las áreas críticas, entendiéndose como tal, las que presentan deficiencias de control. Para identificar estas áreas objetivamente, el auditor desarrollará una matriz que plasme el impacto que ocasionan, en la administración de la entidad (CGE, 2011).

El objetivo es que el auditor desarrolle una estrategia que describa el alcance con el propósito de minimizar el riesgo, hasta un nivel aceptablemente bajo. Con esta estrategia se pretende guiar adecuadamente el desarrollo del plan de auditoría. El auditor debe considerar los resultados de la planeación y hacer uso del conocimiento de la entidad y su entorno, incluyendo el control interno, a fin de que esta estrategia general de auditoría sea efectiva y eficiente. El auditor en base a su juicio profesional determinará la forma y el contenido de esta estrategia (Blanco, 2012).

En el programa de auditoría se describe de forma secuencial y lógica como se deben desarrollar las actividades durante la ejecución del examen; de manera que se puedan cumplir los objetivos propuestos en cada uno de los componentes a examinar; estas actividades se resumen en el memorando de planificación (CGE, 2011). 


\section{Ejecución}

En esta etapa se ejecuta la auditoría siguiendo la planificación desarrollada anteriormente, que permita determinar los resultados de la gestión de la entidad y detectar los hallazgos resultantes que sustentarán los comentarios, las conclusiones y recomendaciones que se incluirán en el informe (CGE, 2011).

El examen consiste en separar los procesos materia de análisis, para conocer su naturaleza y características, sin perder de contexto la relación de las partes entre sí y de estas con el todo (Franklin, 2013). Al respecto, las Normas Ecuatorianas de Auditoría Gubernamental (NEAG) relativas a la ejecución disponen; que al ejecutarse el examen se verificará la observancia de la normativa pertinente a los entes públicos.

Al analizar y validar la información se profundiza en los resultados de las pruebas aplicadas, con la finalidad de formar un juicio profesional que permita emitir una conclusión. En el proceso de ejecución se detectan los hallazgos e identifican las evidencias que fundamentarán las recomendaciones (Sotomayor, 2008).

La etapa de ejecución de la auditoría comienza con la obtención de la evidencia, la misma que permite evaluar los resultados de la gestión y cumplimiento de las leyes para determinar los hallazgos que sustenten los comentarios, conclusiones y recomendaciones que se plasmaran en el informe final.

Las evidencias pueden ser: físicas, documentales, testimoniales y analíticas, y deberán ser evaluadas para el desarrollo de los hallazgos. La evidencia física se obtiene mediante observación directa de las actividades y se presenta por medio de notas, fotografías, o muestras materiales; la documental se obtiene al analizar los documentos y se encuentran en actas, facturas, contratos, registros y todo tipo de documento producto del trabajo; la testimonial son las declaraciones que se obtienen durante el proceso de auditoría y puede ser también los datos derivados de entrevistas o encuestas aplicadas y la analítica son los cálculos, comparaciones y desagregación de la información por componentes (Franklin, 2013).

Una vez obtenida la evidencia suficiente y competente, se aplicarán indicadores de gestión, estos son parámetros utilizados para medir cualitativa y cuantitativamente el nivel 
de observancia de una actividad o proceso, en términos de eficiencia, economía, y efectividad (CGE, 2011).

\section{Papeles de trabajo}

Durante el desarrollo de la auditoría se van elaborando los papeles de trabajo, que fundamentan la elaboración del informe; en el cual se indican dos aspectos significativos que se interrelacionan, estos son: la situación evaluada y las recomendaciones planteadas en el mismo.

El informe es el producto final del trabajo del auditor, quien debe presentarlo de tal manera que el documento se caracterice en contenido y forma, por su calidad, claridad, oportunidad y eficiencia; de manera que queden plasmadas las situaciones encontradas, sobre las cuales deberán tomar decisiones los directivos a quienes se les dirige el mismo (Sotomayor, 2008).

Las (NEAG) señalan, que los papeles de trabajo son los programas escritos que indican el cumplimiento del auditor y la evidencia obtenida por el mismo. Estos permiten fundamentar los hallazgos encontrados, conclusiones y recomendaciones que se entregan en el informe (CGE, 2002).

El auditor debe sustentar documentadamente los asuntos que considere relevantes, esta documentación sirve de soporte para las conclusiones emitidas en el informe y deja evidencia de que la auditoría se llevó a cabo cumpliendo con las normas y regulaciones exigidas; estos papeles de trabajo deben ser completos y detallados de manera que proporcionen una comprensión total de la auditoría.

\section{Comunicación de resultados}

La redacción del informe cumplirá las normas nacionales e internacionales y demás reglamentación, aquí se presenta los hallazgos, conclusiones y recomendaciones. En esta fase se informará permanentemente a la entidad sobre las observaciones encontradas, a fin de obtener justificativos previo a la elaboración del informe final (CGE, 2011). 
El informe contiene las conclusiones a las que llegó el auditor al finalizar el proceso de ejecución, sobre los estados financieros, el control interno, el cumplimiento de la normativa y la gestión. En el documento se indican los detalles de la presunta deficiencia y a quién se ha notificado, en este se debe hacer constar el acuse de recibido de la persona notificada.

\section{Sistema integrado de desarrollo de talento humano}

La aplicación del EE en el sector público puede desarrollarse entre otras áreas, en la de talento humano. En este escenario, Chiavenato (2015) afirma, que el capital intelectual es el activo más valioso de la organización, este activo intangible posee habilidad, conocimiento y experiencia.

Desde la perspectiva de la legislación vigente en el Ecuador para el sector público, la Ley Orgánica del Servicio Público LOSEP, establece que:

El Sistema integrado de desarrollo del talento humano como el conjunto de políticas, normas, métodos y procedimientos orientados a validar e impulsar las habilidades, conocimientos, garantías y derechos de las y los servidores públicos con el fin de desarrollar su potencial y promover la eficiencia, eficacia, oportunidad, interculturalidad, igualdad y la no discriminación en el servicio público para cumplir con los preceptos de esta Ley. (Asamblea Nacional, 2010, p. 28)

El sistema integrado de desarrollo de talento humano está compuesto por cinco subsistemas, estos son: planificación del talento humano, clasificación de puestos, selección de personal, formación y capacitación y evaluación del desempeño. Borrero (2019).

Subsistema de planificación del talento humano. - En este subsistema elaboran la planificación del talento humano, en base a los procesos a ser desarrollados el siguiente año y presentan al Ministerio de Trabajo para su aprobación. Los gobiernos autónomos descentralizados y sus entidades, tendrán su propia planificación anual del talento humano; la que será sometida a su respectivo órgano legislativo (Asamblea Nacional, 2010). 
Subsistema de clasificación de puestos. - Es el conjunto de normas estandarizadas para analizar, describir, valorar y clasificar los puestos en todas las entidades señaladas en el Artículo 3 de esta Ley.

Subsistema de selección de personal. - Es el conjunto de procedimientos, encaminados a evaluar la idoneidad de los aspirantes que reúnan los requerimientos establecidos para el puesto a ser ocupado; garantizando la equidad de género, la interculturalidad y la inclusión de las personas con discapacidad y grupos de atención prioritaria Subsistema de la formación y la capacitación. - La formación es el subsistema de estudios de carrera de nivel superior, que otorga titulación según los conocimientos y que permite a los servidores públicos obtener conocimientos científicos y realizar investigaciones aplicadas al área de prioridad para el país. El subsistema de capacitación está orientado al crecimiento integral del talento humano, a partir de la obtención y actualización de conocimientos.

Subsistema de evaluación del desempeño. - Es el procedimiento armonizado, justo, transparente e imparcial que se orienta a evaluar bajo parámetros objetivos acordes con las funciones, responsabilidades y perfiles del puesto (lbídem).

\section{METODOLOGÍA}

El diseño del presente estudio fue de tipo experimental, ya que se ejecutó un EE para identificar el cumplimiento de los procesos de la Dirección de Desarrollo Institucional y Talento Humano (DDITH); se aplicó el enfoque mixto con un alcance explicativo secuencial, ya que en la primera etapa se recabaron y analizaron datos cualitativos relacionados con el EE y el sistema integrado de talento humano, mientras que, en la segunda fase se recolectaron datos cuantitativos en base a un cuestionario de control interno. Los métodos empleados fueron el histórico - lógico, analítico - sintético, inductivo - deductivo y sistémico.

Según la finalidad el estudio fue transversal, ya que las variables se analizaron en un momento determinado; las técnicas empleadas fueron la encuesta a través de la apli- 
cación de un cuestionario con preguntas cerradas, dirigida a los servidores del departamento de talento humano del Registro de la Propiedad del cantón Cuenca; la entrevista se dirigió a la máxima autoridad de la entidad, por medio de preguntas abiertas con la finalidad de recabar información del área ejecutiva; la observación directa y la revisión documental aportaron en la detección de hallazgos.

\section{RESULTADOS}

De la información recabada luego de la aplicación de las encuestas, se encontraron los siguientes resultados:

- Las autoridades no mantienen controles periódicos de los procesos ejecutados por cada unidad.

- Todo el personal tiene conocimiento de la normativa interna de la institución.

- La entidad utiliza la evaluación de desempeño, para mejorar el rendimiento laboral y tomar medidas correctivas en un $75 \%$.

- La institución no realizó la programación de la capacitación, considerando el perfil y cargo que desempeñan los servidores.

- La entidad ejecuta en un 50\% la programación de la capacitación aprobada.

- No se aplica una adecuada supervisión durante el desarrollo de las funciones, debido a que no se cuenta con una correcta aplicación del sistema de control interno; lo que conlleva a contar con un riesgo alto y un nivel de confianza baja.

- La entidad no cumple al $100 \%$ con los reglamentos emitidos por la Contraloría General del Estado, inobservando así la normativa que le rige.

- Los servidores de la DDITH consideran que la aplicación de un EE como proyecto de investigación, mejoraría el cumplimiento de los procesos administrativos de la unidad.

A partir de los resultados preliminares obtenidos en la fase de diagnóstico, se consideró importante aplicar un EE a la administración de talento humano del Registro de la Propiedad del cantón Cuenca. 


\section{Propuesta}

\section{Esquema de la propuesta}

Figura 1.

\section{El exámen especial como proceso de control}

\section{Fases y aplicación práctica en el sector público}
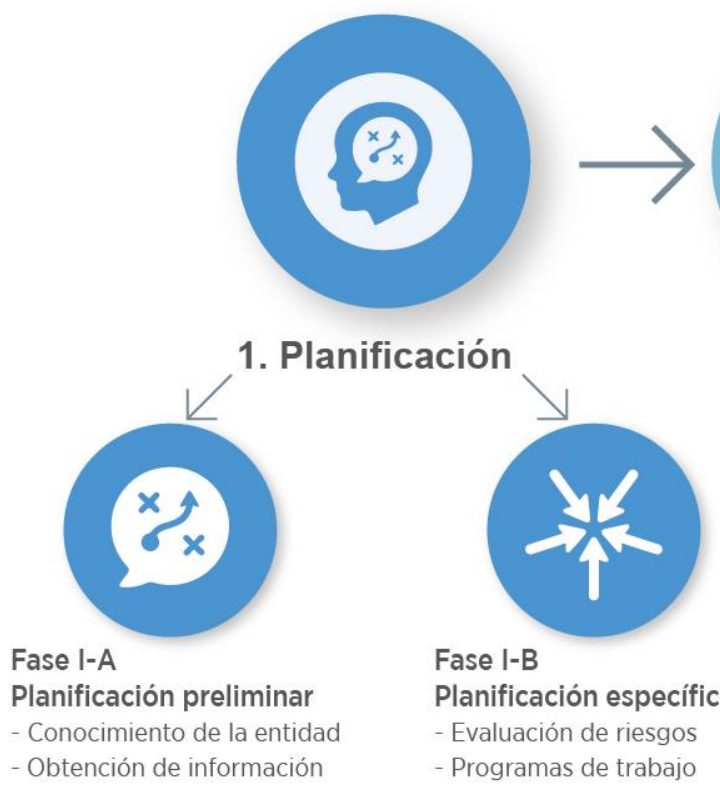

1. Planificación

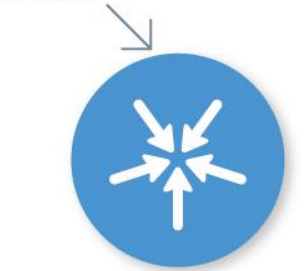

Fase I-B

Planificación específica

- Evaluación de riesgos

- Programas de trabajo

- Evaluación del control interno

- Matriz de enfoque

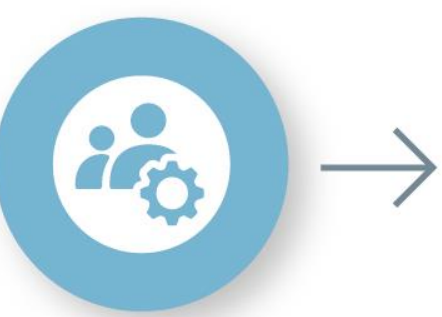

2. Ejecución

- Aplicación de programas

- Papeles de trabajo

- Hojas de hallazgo

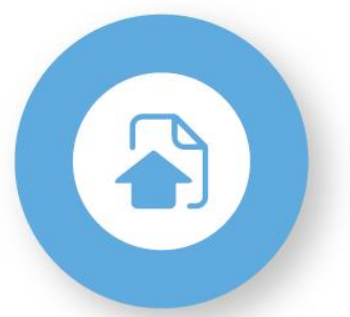

3. Informe

- Informe

- Conclusiones

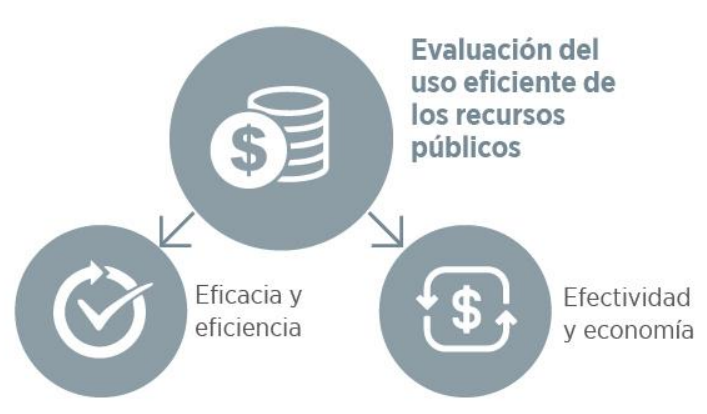

\section{Primera fase del EE}

\section{Planificación preliminar}

Objetivo del EE

Aplicar un EE a la administración de talento humano, con fines de verificación del cumplimiento de los procesos y procedimientos de acuerdo a la normativa vigente.

\section{Alcance del examen}

EI EE comprenderá el análisis de la administración de talento humano del Registro de la Propiedad del cantón Cuenca, por el período comprendido entre el 1 de enero y el 31 de diciembre de 2018. 


\section{Principales disposiciones legales.}

Constitución de la República del Ecuador; Ley Orgánica de la Contraloría General del Estado; Ley Orgánica de Servicio Público y su reglamento; Normas de Control Interno para las Entidades, Organismos del Sector Público y de las personas jurídicas de derecho privado que dispongan de recursos públicos; Código de Trabajo; Normativa interna.

\section{Misión Institucional}

El Registro de la Propiedad del Cantón Cuenca, adscrito al Gobierno Autónomo Descentralizado Municipal del Cantón Cuenca, tiene como misión inscribir en el Registro correspondiente los documentos cuya inscripción exige o permite la Ley, debiendo negarse a inscribir los que no cumple los requisitos de acuerdo a la legislación ecuatoriana vigente, generando un folio personal actualizado con la historia de todos y cada uno de los asientos registrales, para emitir los certificados y/o inscripciones que requiera la ciudadanía usuaria de nuestros servicios, en concordancia con las disposiciones emitidas por la Ley de Registro, Ley del Sistema Nacional del Registro de Datos Públicos, la Ordenanza para la Organización, Administración y Funcionamiento del Registro de la Propiedad del cantón Cuenca y demás leyes correlativas al sector público ecuatoriano. (Brasales, 2013, p. 6)

\section{Visión Institucional}

El Registro de la Propiedad del Cantón Cuenca, adscrito al Gobierno Autónomo Descentralizado Municipal del Cantón Cuenca propende llegar a trabajar en el mediano plazo bajo esquemas jurídico-técnico y tecnológicos actualizados, que le permitan constituirse en pionero a nivel regional y nacional, de la capacidad de respuesta de calidad y eficacia para el usuario de nuestros servicios, por lo tanto el Registro de la Propiedad en el sector público se fundamentará en el mantenimiento de un folio personal y una vez concluido y depurado el mismo nos permitirá la creación de un folio real para poder realizar la interconexión catastro y Registro de la Propiedad. (Brasales, 2013, p. 6) 


\section{Objetivos estratégicos:}

Ámbito Social. - Brindar a todos los usuarios del Registro de la Propiedad del cantón Cuenca, servicios públicos amparados en los principios de eficiencia, calidez, calidad y eficacia, cuidando y precautelando los recursos públicos a base de dar cumplimiento a la normativa del sector público ecuatoriano vigente para todos los frentes de gestión institucional, a saber del: desarrollo institucional y gestión humana, financieros, de recursos materiales y tecnológicos y operativos. (Brasales, 2013, p. 7)

\section{Planificación específica}

\section{Planificación específica}

Tabla 1.

\begin{tabular}{l} 
Examen especial a la administración de talento humano \\
del Registro de la \\
$\qquad$\begin{tabular}{|c|} 
Ref. \\
PP 001
\end{tabular} \\
\hline $\begin{array}{c}01 \text { de enero a } 31 \text { de diciembre de } 2018 \\
\text { Programa de planificación específica }\end{array}$ \\
\hline
\end{tabular}

\section{Objetivo general}

Aplicar un EE a la administración de talento humano, con fines de verificación del cumplimiento de los procesos y procedimientos de acuerdo a la normativa vigente.

Objetivos específicos

- Establecer el enfoque del EE en la DDITH.

- Determinar las estrategias a utilizar en la ejecución del EE.

- Identificar los procesos críticos de la entidad auditada, para examinarlos en la fase de ejecución.

Procedimientos a realizar:

- Evaluación del control interno

- Revisar los expedientes del personal para determinar si se encuentra actualizados. Componentes a analizar:

- Cumplimiento del Acuerdo 045-CG-2016 informe de la presentación de declaraciones patrimoniales en el mes anterior al organismo de control.

- Capacitación del personal. 


\section{Evaluación al sistema de control interno}

Resumen de la evaluación de control interno.

Tabla 2.

\begin{tabular}{|c|c|c|}
\hline \\
\hline Norma técnica aplicada & Respuesta & $\begin{array}{l}\text { Califica- } \\
\text { ción }\end{array}$ \\
\hline \multirow{2}{*}{$\begin{array}{l}\text { La NCI } 200 \text { ambiente de control: comprende los procesos encami- } \\
\text { nados a verificar la integridad y valores éticos, la estructura organiza- } \\
\text { tiva y la competencia profesional de los servidores }\end{array}$} & $\mathrm{Si}$ & 3 \\
\hline & No & 2 \\
\hline \multirow{2}{*}{$\begin{array}{l}\mathrm{NCI} 300 \text { evaluación del riesgo } \\
\text { valoración de los riesgos }\end{array}$} & $\mathrm{Si}$ & 1 \\
\hline & No & 1 \\
\hline \multirow{2}{*}{$\begin{array}{l}\text { La NCI } 400 \text { actividades de control: comprende las actividades de- } \\
\text { talento humano, como: el manual de clasificación de puestos, capaci- } \\
\text { tación y entrenamiento continuo, evaluación de desempeño, rotación } \\
\text { de personal, actuación y honestidad de las servidoras y servidores, } \\
\text { asistencia y permanencia del personal }\end{array}$} & SI & 8 \\
\hline & No & 12 \\
\hline \multirow{2}{*}{$\begin{array}{l}\mathrm{NCI} 500 \text { información y comunicación: se constató el cumplimiento } \\
\text { en procesos de información y comunicación de la entidad }\end{array}$} & $\mathrm{Si}$ & 1 \\
\hline & No & 1 \\
\hline \multirow{2}{*}{$\begin{array}{l}\mathrm{NCl} 600 \text { seguimiento, aquí se evaluaron procedimientos de segui- } \\
\text { miento de actividades, establecidos por la institución }\end{array}$} & $\mathrm{Si}$ & 0 \\
\hline & No & 1 \\
\hline \multirow{3}{*}{$\begin{array}{l}\text { Calificación total CT }=13 \\
\text { Ponderación total PT }=30\end{array}$} & \multicolumn{2}{|c|}{ Calificación } \\
\hline & SI & 13 \\
\hline & NO & 17 \\
\hline
\end{tabular}

De un total de 30 interrogantes planteadas en la aplicación de las encuestas realizadas a los servidores de la institución, se interpretó que se cumplen con las normas de control interno, emitidas por la CGE en 13 procesos de la entidad y por lo contrario se inobserva dicha normativa en 17 procesos restantes de los consultados. 
Matriz de ponderación de la evaluación de control interno.

Tabla 3.

Registro de la Propiedad del cantón Cuenca

Examen especial a la administración de talento humano

Del 01 de enero de 2018 al 31 de diciembre de 2018

\begin{tabular}{|c|c|c|}
\hline \\
\hline \multicolumn{3}{|c|}{ Bajo Moderado } \\
\hline $15 \%-50 \%$ & $51 \%-75 \%$ & $76 \%-95 \%$ \\
\hline $85 \%-50 \%$ & $49 \%-25 \%$ & $24 \%-5 \%$ \\
\hline Alto & Moderado & Bajo \\
\hline \multicolumn{3}{|c|}{$\begin{array}{l}\text { Nivel de riesgo } \\
\text { Valoración }\end{array}$} \\
\hline \multicolumn{3}{|c|}{$\begin{array}{l}\text { NC }=\text { Nivel de confianza } \\
\text { NC }=\frac{\text { calificación total }(C T)}{\text { Ponderación total }(P T)} \times 100 \\
\text { NC }=\frac{\mathbf{1 3}}{\mathbf{3 0}} \times \mathbf{1 0 0} \\
\mathbf{N C}=43,33 \% \text { Bajo }\end{array}$} \\
\hline
\end{tabular}

\begin{tabular}{|l|}
$\begin{array}{l}\text { Determinación de los niveles de } \\
\text { riesgo }\end{array}$ \\
$\mathbf{N R}=$ Nivel de riesgo \\
$\mathbf{N R}=100 \%$ - Nivel de confianza \\
$\mathbf{N R}=100 \%-43,33 \%$ \\
$\mathbf{N R}=\mathbf{5 6 , 6 7} \%$ Alto
\end{tabular}

\section{Evaluación y calificación de los riesgos del EE.}

Matriz de riesgos componente: administración del talento humano Tabla 4

\begin{tabular}{|c|c|c|c|c|c|}
\hline \multirow[b]{2}{*}{$\begin{array}{c}\text { Compo- } \\
\text { nente }\end{array}$} & \multicolumn{2}{|c|}{ Riesgo y su fundamento } & \multirow[b]{2}{*}{$\begin{array}{l}\text { Controles } \\
\text { clave }\end{array}$} & \multicolumn{2}{|c|}{ Enfoque de auditoría } \\
\hline & Inherente & de control & & $\begin{array}{c}\text { Pruebas } \\
\text { sustantivas }\end{array}$ & $\begin{array}{l}\text { Pruebas de } \\
\text { cumpli- } \\
\text { miento }\end{array}$ \\
\hline \multicolumn{6}{|c|}{ Componente: Administración del talento humano } \\
\hline $\begin{array}{l}\text { Planifica- } \\
\text { ción y eje- } \\
\text { cución de } \\
\text { un pro- } \\
\text { grama de } \\
\text { capacita- } \\
\text { ción. }\end{array}$ & $\begin{array}{l}\text { Alto } \\
\text { Falta de un } \\
\text { sistema in- } \\
\text { terno que } \\
\text { permita llevar } \\
\text { una hoja de } \\
\text { vida electró- } \\
\text { nica para fa- } \\
\text { cilitar el re- } \\
\text { gistro y con- }\end{array}$ & $\begin{array}{l}\text { Alto } \\
\text { La DDITH no } \\
\text { mantiene un } \\
\text { adecuado } \\
\text { control sobre } \\
\text { la ejecución } \\
\text { del programa } \\
\text { de capacita- } \\
\text { ción. }\end{array}$ & $\begin{array}{l}\text { Certificados de } \\
\text { asistencia y } \\
\text { aprobación de } \\
\text { los cursos pla- } \\
\text { nificados. }\end{array}$ & $\begin{array}{l}\text { Verificar que } \\
\text { se esté eje- } \\
\text { cutando el } \\
\text { plan de capa- } \\
\text { citación apro- } \\
\text { bado por la } \\
\text { máxima auto- } \\
\text { ridad. }\end{array}$ & $\begin{array}{l}\text { Verificar que } \\
\text { se haya ela- } \\
\text { borado el } \\
\text { plan de capa- } \\
\text { citación para } \\
\text { el período } \\
\text { evaluado. }\end{array}$ \\
\hline
\end{tabular}




\begin{tabular}{|c|c|c|c|c|c|}
\hline & $\begin{array}{l}\text { trol de la ca- } \\
\text { pacitación re- } \\
\text { cibida. }\end{array}$ & & & & \\
\hline $\begin{array}{l}\text { Presenta- } \\
\text { ción men- } \\
\text { sual del re- } \\
\text { porte de de- } \\
\text { claraciones } \\
\text { patrimonia- } \\
\text { les a la CGE }\end{array}$ & $\begin{array}{l}\text { Bajo } \\
\text { Baja rotación } \\
\text { de servidores } \\
\text { públicos. }\end{array}$ & $\begin{array}{ll}\text { Alto } & \\
\text { Ausencia de } \\
\text { control in- } \\
\text { terno. }\end{array}$ & $\begin{array}{l}\text { Verificar el } \\
\text { cumplimiento } \\
\text { del Acuerdo } \\
045 \text { - CG - } \\
2016 \text {. }\end{array}$ & $\begin{array}{l}\text { Verificar la } \\
\text { oportuna en- } \\
\text { trega de in- } \\
\text { formación al } \\
\text { organismo de } \\
\text { control. }\end{array}$ & $\begin{array}{l}\text { Verificar si se } \\
\text { cumplió con } \\
\text { la normativa } \\
\text { establecida } \\
\text { para la pre- } \\
\text { sentación del } \\
\text { reporte de } \\
\text { declaracio- } \\
\text { nes patrimo- } \\
\text { niales. }\end{array}$ \\
\hline
\end{tabular}

Programa de auditoría.

Tabla 5.

\section{Examen especial a la administración de talento humano del Registro de la Propiedad del cantón Cuenca 1 de enero 2018 al 31 de diciembre de 2018 Programa de auditoría}

\begin{tabular}{|l|l|l|l|}
\hline Entidad: & $\begin{array}{l}\text { Registro de la Propiedad del cantón } \\
\text { Cuenca. }\end{array}$ & Alcance & 2018-01-01 al 2018-12-31 \\
\hline Provincia & Azuay & Auditora & $\begin{array}{l}\text { Diana Paulina Figueroa } \\
\text { Hurtado }\end{array}$ \\
\hline Ciudad & Cuenca & Fecha & 9 de enero de 2019 \\
\hline
\end{tabular}

Componente: administración de talento humano

Objetivo general: Aplicar un EE a la administración de talento humano, con fines de verificación del cumplimiento de los procesos y procedimientos de acuerdo a la normativa vigente.

\begin{tabular}{|c|l|c|c|c|c|}
\hline No. & Procedimientos & $\begin{array}{c}\text { Tiempo } \\
\text { estimado }\end{array}$ & $\begin{array}{c}\text { Reali- } \\
\text { zado por }\end{array}$ & Fecha & $\begin{array}{c}\text { REF/ } \\
\text { PT }\end{array}$ \\
\hline $\mathbf{1}$ & $\begin{array}{l}\text { Pruebas de cumplimiento } \\
\text { plan de capacitación para el período evaluado }\end{array}$ & & DPFH & & \\
\hline
\end{tabular}


Revista Arbitrada Interdisciplinaria KOINONIA

Año IV. Vol IV. N². Edición especial 2019

Hecho el depósito de Ley: FA2016000010

ISSN: 2542-3088

FUNDACIÓN KOINONIA (F.K). Santa Ana de Coro. Venezuela.

Diana Paulina Figueroa Hurtado; Cecilia Ivonne Narváez Zurita; Juan Carlos Erazo Álvarez

\begin{tabular}{|c|c|c|}
\hline 2 & $\begin{array}{l}\text { Verificar si se cumplió con la normativa esta- } \\
\text { blecida para la presentación del reporte de de- } \\
\text { claraciones patrimoniales. }\end{array}$ & DPFH \\
\hline & Pruebas sustantivas & DPFH \\
\hline 1 & $\begin{array}{l}\text { Verificar que se esté ejecutando el plan de ca- } \\
\text { pacitación aprobado por la máxima autoridad. }\end{array}$ & DPFH \\
\hline 2 & $\begin{array}{l}\text { Verificar la oportuna entrega de información al } \\
\text { organismo de control. }\end{array}$ & DPFH \\
\hline
\end{tabular}

\section{Segunda fase del EE - Ejecución}

\section{Hoja de hallazgo.}

Tabla 6.

Título: La entidad no cumplió con la programación de capacitación aprobada por la máxima autoridad.

Condición: La entidad no cumple en su totalidad con el programa de capacitación.

\section{Criterio:}

200-03 - Políticas y prácticas de talento humano.

"...La administración del talento humano, constituye una parte importante del ambiente de control con el papel esencial de fomentar un ambiente ético desarrollando el profesionalismo y fortaleciendo la transparencia en las prácticas diarias. Esto se hace visible en la ejecución de los procesos de planificación, clasificación, reclutamiento y selección de personal, capacitación, evaluación del desempeño y promoción y en la aplicación de principios de justicia y equidad, así como el apego a la normativa y marco legal que regulan las relaciones laborales...".

200-06 - Competencia profesional.

"...Las servidoras y servidores de los organismos, entidades, dependencias del sector público y personas jurídicas de derecho privado que dispongan de recursos públicos, mantendrán un nivel de competencia que les permita comprender la importancia del desarrollo, implantación y mantenimiento de un buen control interno y realizar sus funciones para poder alcanzar los objetivos y la misión de la entidad.

Los directivos de la entidad, especificarán en los requerimientos de personal, el nivel de competencias necesario para los distintos puestos y tareas a desarrollarse en las áreas correspondientes. Así mismo, los programas de capacitación estarán dirigidos a mantener los niveles de competencia requeridos..."

Causa: No se aplicó un sistema de control interno, que permita verificar la continua ejecución del programa de capacitación aprobado.

Efecto: No se cumplió con la programación de capacitación, lo que dificulta mantener un personal capacitado para desarrollar de manera óptima su trabajo 
Conclusión: La falta de capacitación de los servidores, no permite mantener el grado de competencia requerida en el desarrollo de sus actividades.

Hoja de hallazgo.

Tabla 7.

Título: La Dirección de Desarrollo Institucional y Talento Humano no presentó información a la CGE con relación a los contratos y nombramientos celebrados.

Condición: De la información solicitada al Registro de la Propiedad del Cantón Cuenca, se evidenció que la DDITH no presentó los informes mensuales a la Contraloría General del Estado, sobre los contratos y nombramientos celebrados el mes anterior; así como del cese de funciones producido en el mismo período.

Criterio:

Ley Orgánica de la Contraloría General del Estado

Art. 77.- Máximas autoridades, titulares y responsables.

2. Autoridades de las unidades administrativas y servidores:

“...a) Contribuir a la obtención de los fines institucionales y administrar en el área que les competa, los sistemas a que se refiere el literal a) del numeral anterior..."

Normas de control interno emitidas por la CGE

100-03 - Responsables del control interno.

"...Las servidoras y servidores de la entidad, son responsables de realizar las acciones y atender los requerimientos para el diseño, implantación, operación y fortalecimiento de los componentes del control interno de manera oportuna, sustentados en la normativa legal y técnica vigente...".

Reglamento para la declaración, presentación y registro de las declaraciones patrimoniales juradas, otorgadas electrónicamente a través de la página web de la contraloría general del estado.

Artículo 7.- De la información remitida por las Unidades de Administración del Talento Humano de las instituciones del sector público.

.... Las Unidades de Administración del Talento Humano o quien hiciera sus veces, remitirán a la Contraloría General del Estado en los diez primeros días de cada mes, el detalle de los servidores/as a quienes les correspondía efectuar las declaraciones periódicas, así como a los que se les otorgó nombramientos, contratos o cese de funciones celebrados el mes inmediato anterior..." -;- "...Las Unidades de Administración del Talento Humano o quien hiciera sus veces, deberán velar por el cumplimiento de esta disposición, de no hacerlo, será considerado como falta grave y sancionado en los términos que prevé la Ley Orgánica del Servicio Público....".

Causa: El jefe de la DDITH inobservó la disposición de remitir a la Contraloría General del Estado en los diez primeros días de cada mes la información de los servidores que deben presentar las declaraciones juradas de bienes y de los contratos y nombramientos celebrados el mes anterior.

Efecto: Lo que ocasionó que el Organismo de Control no cuente con la información necesaria para realizar el control posterior.

Conclusión: El jefe de la DDITH no cumplió con la normativa vigente emitida por la Contraloría General del Estado. 


\section{Tercera fase del EE - Informe final}

Se efectúo el EE a la administración del talento humano del Registro de la Propiedad del cantón Cuenca, por el período comprendido del 1 de enero al 31 de diciembre de 2018, la acción de control se efectúo de acuerdo con las NEAG emitidas por la CGE, Normativa del Ministerio de Trabajo, Normativa de la Contraloría General del Estado y normativa interna de la institución; estas normas requieren que el EE sea planificado y ejecutado para obtener certeza de que la documentación examinada no contenga errores significativos, igualmente que los procesos evaluados se hayan desarrollado de conformidad con las disposiciones legales vigentes. Se evalúo el sistema de control interno, aplicando un cuestionario basado en el enfoque COSO I (Comité de organizaciones auspiciantes), con lo que se pudo determinar que la entidad tiene un nivel de riesgo alto; por lo que se recomienda a los directivos y a todo el personal supervisar y cumplir con las Normas de Control Interno emitidas por la Contraloría General del Estado y que rigen al sector público. Se evalúo la eficiencia y eficacia en la consecución de las metas programadas por la entidad, determinándose que la entidad no cumple con lo planificado, respecto a la capacitación de su personal; así mismo se pudo constatar que no cumple con lo establecido en el Acuerdo 045-CG-2016 Informe de la presentación de declaraciones patrimoniales en el mes anterior al organismo de control.

\section{CONCLUSIONES}

- El EE como proceso de control del uso debido de los recursos públicos, permitió evaluar el control interno aplicado en la unidad de análisis y determinar el cumplimiento de las normas de control interno emitidas por la CGE que rigen para el sector público y privado que dispongan de recursos públicos.

- La evaluación de control interno permitió determinar que la entidad cuenta con un nivel de riesgo alto, debido a la falta de aplicación de un correcto sistema de control interno en algunos de los componentes del enfoque coso I, con lo que se pudo 
concluir que la entidad no está cumpliendo con su objetivo estratégico de cumplir con la normativa del sector público ecuatoriano vigente.

- Al ejecutar el examen se pudo identificar el cumplimiento o inobservancia de la normativa, recopilar la evidencia suficiente, competente y pertinente que fundamente los hallazgos encontrados, los mismos que se plasmaron en el informe final.

\section{REFERENCIAS CONSULTADAS}

1. Asamblea Nacional. (06 de 10 de 2010). De la Administración Técnica del Talento Humano. Ley Orgánica del Servicio Público. Quito, Pichincha, Ecuador.

2. Atehortúa Hurtado, F. A., Bustamante Vélez, R. E., \& Valencia de los Ríos, J. A. (2008). Sistema de Gestión Integral. Colombia: Editorial Universidad de Antioquia.

3. Borrero, R. (2019). Procesos de gestión del talento humano en el sector educativo gerencial de Colombia. Revista Arbitrada Interdisciplinaria Koinonía, 4(7), 293-307. doi:http://dx.doi.org/10.35381/r.k.v4i7.206

4. Blanco, L. Y. (2012). Auditoría Integral, Nomas y Procedimientos. Bogotá: Ecoe Ediciones.

5. Brasales, J. A. (1 de Agosto de 2013). Resolución No. 23 - 2013. Estatuto Orgánico de Estructura Organizacional de Gestión por Procesos del Registro de la Propiedad del Cantón Cuenca. Cuenca, Azuay, Ecuador: Registro de la Propiedad del Cantón Cuenca. Obtenido de Página institucional: http://www.regprocue.gob.ec/registropropiedad/content/visión-y-misión

6. CGE. (10 de Octubre de 2002). Acuerdo 019- CG - 2002. Normas Ecuatorianas de Auditoría Gubernamental. San Francisco de Quito, Pichincha, Ecuador.

7. CGE. (19 de 06 de 2003). Acuerdo 012-CG-2003. Manual General de Auditoría Gubernamental. Quito, Pichincha, Ecuador.

8. CGE. (20 de 12 de 2011). Acuerdo 047-CG-2011. Guía metodológica para auditoría de gestion. Quito, Pichincha, Ecuador. 
9. Chiavenato, I. (2007). Administración de Recursos Humanos. México: McGrawHill / Interamericana Editores, S.A. DE C.V. Recuperado el 26 de 06 de 2018.

10. Chiavenato, I. (2015). Gestión de Talento Humano. México: McGraw-Hill / Interamericana Editores, S.A. DE C.V.

11. Congreso Nacional. (12 de 06 de 2002). Ley Orgánica de la Contraloría General del Estado. Sistema de Control Externo. San Francisco de Quito, Pichincha, Ecuador.

12. Constitución de la República del Ecuador 2008. (20 de Octubre de 2008). Constitución de la República del Ecuador . Administración pública. Quito, Pichincha, Ecuador.

13. Estupiñan, G. R. (2015). Administración de Riesgos E.R.M. y la Auditoría Interna. Bogotá: Ecoe Ediciones.

14. Fonseca, O. (2007). Auditoría Gubernamental Moderna. Lima: Enlace Gubernamental S.A.C.

15. Franklin, E. B. (2013). Auditoría Administrativa (Tercera Edición ed.). México: Pearson Educación. Recuperado el 11 de 06 de 2018.

16. Instituto Mexicano de Contadores Públicos. (2002). Actualización Contable. Norma Internacional de Auditoría 4. Planeación Sección 300. Quito, Pichincha, Ecuador: Corporación Edi Ábaco Cia. Ltda.

17. INTOSAI. (2013). ISSAI 100. Principios Fundamentales de Auditoría del Sector Público. Viena, Austria.

18. Mantilla, S. A. (2018). Auditoría del Control Interno. Bogotá: ECOE Ediciones.

19. Norma Internacional ISO 19011. (15 de 11 de 2011). Norma Internacional ISO 19011. Directrices para la auditoría de Sistemas de Gestión. Suiza: ISO copyright office.

20. Organización Internacional de Entidades Fiscalizadoras Superiores (INTOSAI). (2013). ISSAI 100. Principios Fundamentales de Auditoría del Sector Público. Viena, Austria. 
21. Portal, J. M. (2016). Control interno e integridad: elementos necesarios para la gobernanza pública. Red de Revistas Científicas de América Latina y el Caribe, España y Portugal, 12.

22. Pucha, T. R., Narváez, Z. C., Erazo, Á. J., \& Torres, P. M. (2019). Examen Especial a las operaciones administrativas y financieras de la cuenta transitoria viáticos, movilización y subsistencia del personal de la coordinación provincial de pensiones del IESS - Loja en el período 1 de enero al 31 de diciembre de 2017. Visionario Digital.

23. Sotomayor, A. A. (2008). Auditoría Administrativa. Mèxico, D.F.: McGRAWHILInteramericana Editores, S.A. de C.V.

24. Tapia, I. C., Guevara, R. E., Castillo, P. S., Rojas, T. M., \& Salomón, D. L. (2016). Fundamentos de Auditoría. Mèxico: Instituto Mexicano de Contadores Públicos.

25. Whittington, O. R., \& Pany, k. (2005). Principios de Auditoría. México: McGrawHill/Interamericana Editores, S.A. DE C.V.

\section{REFERENCES CONSULTED}

1. National Assembly. (06 of 10 of 2010). From the Technical Administration of Human Talent. Organic Law of Public Service. Quito, Pichincha, Ecuador.

2. Atehortúa Hurtado, F. A., Bustamante Vélez, R. E., \& Valencia de los Ríos, J. A. (2008). Integral Management System Colombia: Editorial University of Antioquia.

3. Borrero, R. (2019). Human talent management processes in the management education sector of Colombia. Interdisciplinary Arbitrated Review Koinonía, 4 (7), 293-307. doi: http: //dx.doi.org/10.35381/r.k.v4i7.206

4. Blanco, L. Y. (2012). Integral Audit, Nominations and Procedures. Bogotá: Ecoe Editions.

5. Brasales, J. A. (August 1, 2013). Resolution No. 23 - 2013. Organic Statute of Organizational Structure of Process Management of the Land Registry of Canton Cuenca. Cuenca, Azuay, Ecuador: Land Registry of Canton Cuenca. Obtained from Institutional page:

http://www.regprocue.gob.ec/registropropiedad/content/visión-y-mission 
6. CGE. (October 10, 2002). Agreement 019- CG - 2002. Ecuadorian Government Audit Standards. San Francisco de Quito, Pichincha, Ecuador.

7. CGE. (19 of 06 of 2003). Agreement 012-CG-2003. General Manual of Government Audit. Quito, Pichincha, Ecuador.

8. CGE. (20 of 12 of 2011). Agreement 047-CG-2011. Methodological guide for management audit. Quito, Pichincha, Ecuador.

9. Chiavenato, I. (2007). Human resources management. Mexico: McGraw-Hill / Interamericana Editores, S.A. OF C.V. Retrieved on June 26, 2018.

10. Chiavenato, I. (2015). Human Talent Management. Mexico: McGraw-Hill / Interamericana Editores, S.A. OF C.V.

11. National Congress. (12 of 06 of 2002). Organic Law of the Comptroller General of the State. External Control System San Francisco de Quito, Pichincha, Ecuador.

12. Constitution of the Republic of Ecuador 2008. (October 20, 2008). Constitution of the Republic of Ecuador. Public administration. Quito, Pichincha, Ecuador.

13. Estupiñan, G. R. (2015). Risk Management E.R.M. and Internal Audit. Bogotá: Ecoe Editions.

14. Fonseca, O. (2007). Modern Government Audit. Lima: Enlace G Gobiernomental S.A.C.

15. Franklin, E. B. (2013). Administrative Audit (Third Edition ed.). Mexico: Pearson Education. Retrieved on 11 from 06 of 2018.

16. Mexican Institute of Public Accountants. (2002). Accounting Update. International Audit Standard 4. Planning Section 300. Quito, Pichincha, Ecuador: Corporación Edi Ábaco Cia. Ltda.

17. INTOSAI. (2013). ISSAI 100. Fundamental Principles of Public Sector Audit. Vienna, Austria

18. Mantilla, S. A. (2018). Internal Control Audit. Bogotá: ECOE Editions. 
19. International Standard ISO 19011. (15 of 11 of 2011). International Standard ISO 19011. Guidelines for the audit of Management Systems. Switzerland: ISO copyright office.

20. International Organization of Supreme Audit Institutions (INTOSAI). (2013). ISSAI 100. Fundamental Principles of Public Sector Audit. Vienna, Austria

21. Portal, J. M. (2016). Internal control and integrity: elements necessary for public governance. Network of Scientific Journals of Latin America and the Caribbean, Spain and Portugal, 12.

22. Pucha, T. R., Narváez, Z. C., Erazo, Á. J., \& Torres, P. M. (2019). Special Examination of the administrative and financial operations of the transitory account, travel and subsistence of staff of the provincial pension coordination of the IESS - Loja in the period from January 1 to December 31, 2017. Digital Visionary.

23. Sotomayor, A. A. (2008). Administrative audit. Mexico, D.F .: McGRAWHILInteramericana Editores, S.A. from C.V.

24. Tapia, I. C., Guevara, R. E., Castillo, P. S., Rojas, T. M., \& Salomón, D. L. (2016). Fundamentals of Audit. Mexico: Mexican Institute of Public Accountants.

25. Whittington, O. R., \& Pany, k. (2005). Principles of Audit. Mexico: McGraw-Hill / Interamericana Editores, S.A. OF C.V. 